\title{
Non-destructive evaluation by terahertz spectroscopy for penetration of acid solutions into epoxy resin
}

\author{
M. Kusano ${ }^{1 *}$, M. Kubouchi ${ }^{2}$, D. S. Bulgarevich ${ }^{1}$, M. Shiwa $^{1}$ \\ ${ }^{1}$ National Institute for Materials Science, 1-2-1 Sengen, Tsukuba, 305-0047 Ibaraki, Japan \\ ${ }^{2}$ Tokyo Institute of Technology, 2-12-1, O-okayama, Meguro, 152-8550 Tokyo, Japan
}

Received 18 April 2016; accepted in revised form 27 June 2016

\begin{abstract}
Epoxy resins are used as high-performance thermosetting linings to protect substrates under corrosive environments. However, in a severe corrosive chemical solution, such protective layers may degrade with long time due to penetrations of solvent and solute molecules into resin network. In this regard, the terahertz time-domain spectroscopy (THz-TDS) is a promising tool for non-destructive evaluation of the penetrant amounts due to high transparency of such plastic materials and high sensitivity to the molecular vibrations in terahertz spectral range. In this work, the complex refractive indexes $n$ and $\kappa$ of epoxy specimens were measured after immersion into sulfuric acid solutions and compared with penetrated mass fractions of water and acid ions. It was found that $n$ and $\kappa$ depended linearly with water and sulfuric acid mass fraction in specimens, and $\kappa$ of sulfuric acid immersed specimens was lager at higher frequency. While the calculated $\Delta \kappa$ agreed well with THz-TDS measurement by THz-TDS, the calculated $\Delta n$ was higher than the measurement. The difference may be attributed to the water and sulfuric states in the specimen.
\end{abstract}

Keywords: thermosetting resins, terahertz spectroscopy, complex refractive index, epoxy resin, sulfuric acid

\section{Introduction}

Epoxy resins have excellent properties such as adhesion, corrosion resistance, and mechanical strength. Therefore, they are classified as high-performance thermosetting resins for coatings and linings. In particular, the amine-cured epoxies can cure at ambient conditions and are known as the most common coatings for metal equipment and concrete constructions under corrosive environments [1]. When these coatings are used in chemical solutions such as process fluids in chemical plants and sewage water in communities, the water and solute molecules gradually penetrate into the epoxy resins, especially under low $\mathrm{pH}$ acid conditions [2-7]. After the penetrants reach the interface between the resin coating and the substrate, the substrate can be corroded by the penetrants. In order to use these protective materials for a long-term, it is important to non-destructively evaluate the amounts and depths of the penetrants in the polymer coatings/lining. However, there are only a few non-destructive testing (NDT) methods that can measure the amounts of water and solutes inside coatings/linings. For example, the ultrasonic test (UT), which is one of the most common NDT methods, can measure the variation of the sound velocity in degraded samples [8]. However, it is still difficult to estimate the penetrants in resin by UT. On the other hand, with portable FT-IR Attenuated Total Reflection (ATR) spectroscopy, it is possible to non-destructively detect water, sulfate, or other penetrant in plastics [9, 10], but the depth of FT-IR ATR measurement into plastics is only submicron at most $[11,12]$, which is much smaller than typical thickness of resin coatings/linings (typically more than $1 \mathrm{~mm}$ ). Therefore, 
FT-IR ATR spectroscopy is also not suitable for the NDT method of coatings/linings.

The terahertz frequency ranges between 0.1 and $10 \mathrm{THz}$ correspond to the spectral region between microwave and far-IR. Due to the progress in terahertz time-domain spectroscopy (THz-TDS), it has received an increased interest during the past decade. The main advantages of THz-TDS for NDT studies are the high penetration of $\mathrm{THz}$ electromagnetic waves into polymers [13-18], high sensitivity to the inter/intra molecular vibrations (especially for water) [18-26], and ability to estimate the materials dielectric properties without direct contact [27-29].

The THz spectra of various thermoplastics [13-15] and thermosets $[16,17]$ measured by THz-TDS were reported. In contrast with FT-IR spectra, the $\mathrm{THz}$ spectra shows a few clear features to distinguish polymer molecular structures. However, THz spectroscopy can be useful for monitoring the glass transition of the polymer because the polymer chain motion above and below the glass transition temperature affects the THz refractive index [14]. In addition, owing to the significant difference of dielectric properties between polymers and other materials, spectral features of inclusions such as inorganic fillers [15] and carbon nanotubes [17] in polymer materials were also recognized in the $\mathrm{THz}$ spectra.

The dielectric properties of liquid water, aqueous solutions, and water in materials were also studied with to understand hydrogen bonding state i.e. 'free water' and 'bound water' [23-26]. Although free and bound water was experimentally studied by FT-IR [10, 30, 31], NMR [32], thermomechanical analysis [31, 32] etc., it is still a highly controversial subject. Since the interactions of the bound water and free water show slightly different dielectric properties [14, 20, 22 $25,29]$, THz-TDS could have an advantage to distinguish these two states of water. In fact, the $\mathrm{THz}$ dielectric spectra of bulk water is composed of several spectral peaks related to the different relaxation times and intermolecular stretching vibration of water [23, 24].

The detection and imaging of liquid water and moisture in materials by $\mathrm{THz}$ spectroscopy had been previously studied and various applications had been demonstrated on medical, agricultural, food, and other products $[20,27]$. Note that water molecules in material can also exist as both 'free' and 'bound' water and it is difficult to separate experimentally the contributions from bound and free water to the $\mathrm{THz}$ spectra [20]. In certain cases, the assumption that only bound water exists in a material makes it possible to measure its content $[18,20]$. For example, the amounts of water in polyamide and wood plastic composites were estimated with THz-TDS by measuring the complex refractive index as a function of water content [18]. In the case of wood materials in humid environment, the real and imaginary parts of the complex dielectric function of the samples strongly correlated with density and moisture content [21].

As described above, the interpretation of $\mathrm{THz}$ spectra for penetrants in polymer still remains unclear but if penetrants such as sulfuric acid in polymer affect $\mathrm{THz}$ spectra, THz spectroscopy may help to nondestructively monitor polymer degradation by corrosive solution penetration and evaluate the penetrant content in polymer. To the best of our knowledge, there is no study with $\mathrm{THz}$ spectroscopy on penetrants in polymeric materials except for water.

In this work, the epoxy resin immersed in sulfuric acid aqueous solutions was observed by THz-TDS. It was shown that the imaginary part of the complex refractive index in $\mathrm{THz}$ range was correlated linearly and independently with the amounts of water and sulfuric acid in epoxy resin.

\section{Experimental}

\subsection{Specimens and immersion environments}

Epoxy resin (EP) specimens were prepared in the following manner. Firstly, a bisphenol-A-type epoxy resin $\left(\right.$ EPOMIK $^{\circledR}$ R140, Mitsui Chemicals, Inc., Japan) was mixed with equivalent ratio of curing agent (polyether amine, JEFFAMINE ${ }^{\circledR}$ D-230, Huntsman Corporation, Japan). The mixture was evacuated in a deforming device to remove air bubbles, then it was cast into a metal mold and placed into an oven at appropriate temperature until curing was completely finished. After that, the broad cured EP plate $2 \mathrm{~mm}$ thick was taken out from the mold and cut into specimens with $30 \times 30 \mathrm{~mm}$ dimensions.

Ion-exchanged water and $2.0 \mathrm{M}$ sulfuric acid aqueous solutions (Kanto Chemical Co., Inc., Japan) were used as environmental solutions. The beakers with such solutions were set in a water bath at $70^{\circ} \mathrm{C}$. The EP specimens were immersed into solutions and then taken out periodically. Then, they were wiped off 
with paper towel to measure their mass by an analytical balance, thickness by a micrometer, and transmission spectrum by THz-TDS. After these measurements, the specimens were dried in air at $50^{\circ} \mathrm{C}$ until their masses remained constant for a day. Then, their mass, thickness, and transmission spectrum were measured again.

\subsection{Degradation analysis}

In acid aqueous solutions, the water and acid could penetrate into the immersed resin specimen with time [4-6]. In order to evaluate the amounts of penetrants, the mass of the specimen was measured soon after removal from the solution ( $\left.m_{\mathrm{wet}}\right)$ and after drying in air at $50{ }^{\circ} \mathrm{C}\left(m_{\text {dry }}\right)$. The wet $\left(M_{\text {wet }}\right)$ and dry $\left(M_{\text {dry }}\right)$ mass fractions were determined by Equations (1) and (2):

$$
M_{\mathrm{wet}}=\frac{m_{\mathrm{wet}}-m_{0}}{m_{0}}
$$

and

$$
M_{\mathrm{dry}}=\frac{m_{\mathrm{dry}}-m_{0}}{m_{0}}
$$

where $m_{0}$ was the initial mass.

After drying, the specimens were cut and their cross sections were analyzed by energy dispersive X-ray spectroscopy, EDS (equipped on JSM-6510 LA, JEOL Ltd., Japan). The sulfur element mapping on the cross section provides the penetration depth of sulfuric acid.

\subsection{Terahertz time-domain spectroscopy}

The THz-TDS instrument with bound IR-spectra software (IRS-2000, Aispec Co. Ltd., Japan) was used

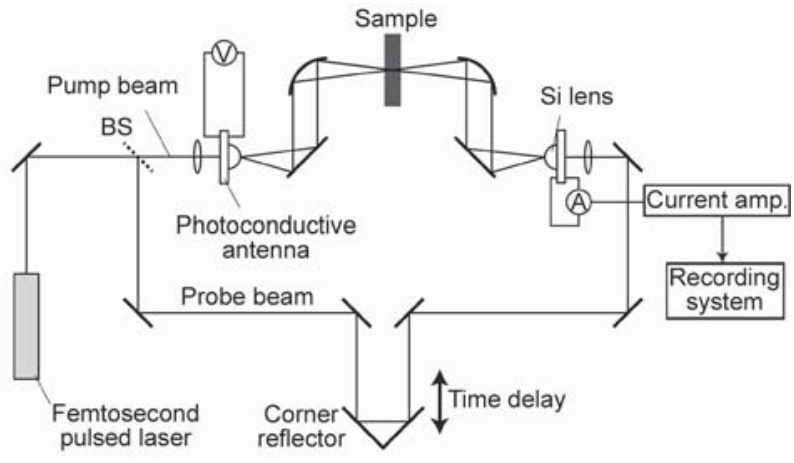

Figure 1. The scheme of the THz-TDS system used in experiments to measure transmission spectra of the EP specimens. Figure 1 displays typical transmission-type THz-TDS setup [28, 29]. In this optical arrangement, the modulated femtosecond laser pulse of about $10 \mathrm{fs}$ duration at a wavelength of $780 \mathrm{~nm}$ is split into the pump and probe beams. The pump beam helps to generate ultrashort current flow inside illuminated photoconductive substrate in the gap between biased antenna electrodes (a low-temperature-grown $\mathrm{Ga}-\mathrm{As}$ film). Then, the broadband $\mathrm{THz}$ radiation from this gap is reshaped spectrally and redirected spatially by antenna electrodes and optical elements towards sample. Finally, $\mathrm{THz}$ radiation arrives to the second identical photoconductive antenna (PCA) at the same time with the laser probe beam. As a result, the modulated current is generated at the PCA detector gap and registered with lock-in amplifier. In order to record the time-domain signal of the pump beam, the optical length of the probe beam is changed by moving a corner reflector as indicated by an arrow in Figure 1 (in other words, the time the probe beam arrives at the PCA is changed). As a consequence, $\mathrm{THz}$ waveform can be digitized at fine time intervals.

The $\mathrm{THz}$ waveform transmitted through specimens was measured under ambient conditions $\left(24^{\circ} \mathrm{C}\right.$, $\sim 40 \% \mathrm{RH}$ ). In addition, the waveform without specimens at the same ambient conditions was also measured as a reference every time. Since the THz beam focuses on sample and the beam width at sample is less than $10 \mathrm{~mm}$, the dimensions of the EP specimens were large enough not to consider whether the edge of the specimen affected the transmission spectra in this study. The measured waveforms were converted into the frequency domain $\mathrm{THz}$ transmission spectrum and phase delay data by fast Fourier transform (FFT). From the spectrum and phase delay data of specimen and reference, IR-spectra software calculates the complex refractive indexes $(\tilde{n}=n-i \kappa)$ of the EP specimens in THz range. The mathematical algorithm is described in detail elsewhere [28, 29]. Since the transmittance of electromagnetic waves through a $2 \mathrm{~mm}$ thick epoxy specimen rapidly decreases with increasing frequency above $1 \mathrm{THz}$, the effective frequency range is limited up to $1 \mathrm{THz}$. For the same reason, the range is also limited to above $0.2 \mathrm{THz}$ 


\section{Results}

\subsection{EP immersed in water}

Firstly, the EP specimen after immersion in ion-exchanged water was observed. Figure 2 shows $M_{\text {wet }}$ of the EP specimen with immersion times in ion-exchanged water. As shown in this figure, $M_{\text {wet }}$ increases proportionally with square root of immersion time due to penetration of the water molecules into EP network by Fick's law of diffusion [2, 3]. After about 900 hours ( 37.5 days), the $M_{\text {wet }}$ reached to a saturation level. The mass after drying in air at $50^{\circ} \mathrm{C}, m_{\text {dry }}$ returns to the initial value, $m_{0}$ because water molecules in EP have completely evaporated.

Figure 3 shows the spectral change of $n$ and $\kappa$ of EP through water immersion from 0 to 49 days. Both of them are shifted vertically and incrementally with immersion time. After drying, such spectra return to their initial values ( 0 days). In order to correlate the complex refractive index with water content in EP, $n$ and $\kappa$ at 0.4 and $0.6 \mathrm{THz}$ as representative values are plotted with $M_{\text {wet }}$. As shown in Figure 4, both $n$

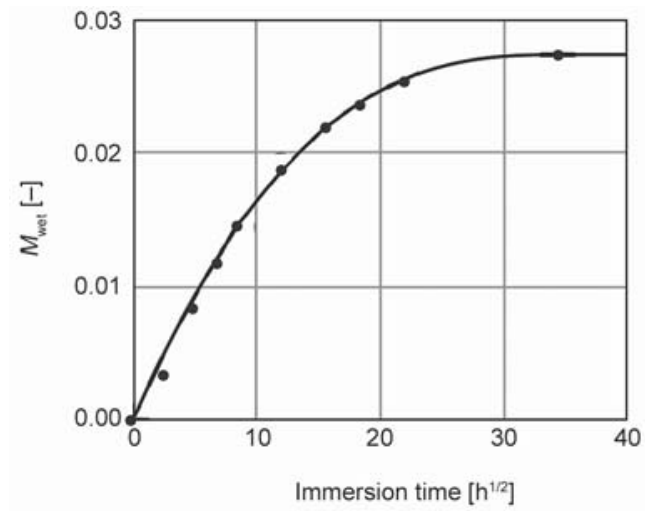

Figure 2. The wet mass fraction of the EP specimen with immersion times in ion-exchanged water

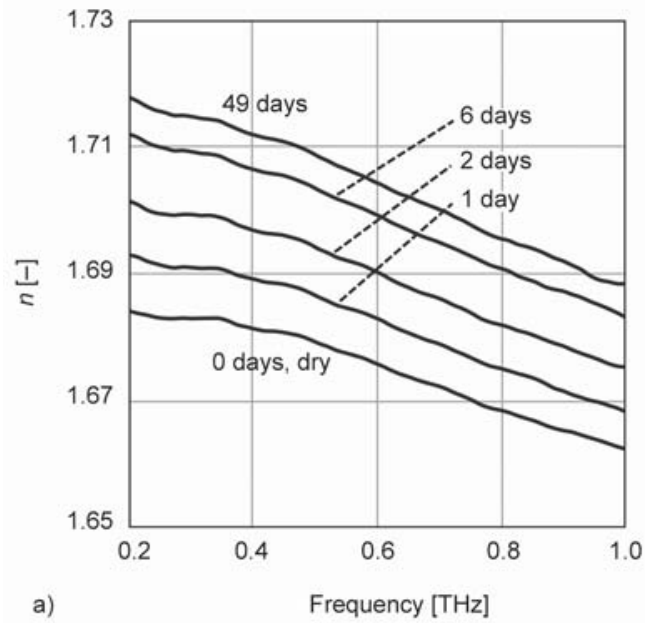

and $\kappa$ at 0.4 and $0.6 \mathrm{THz}$ increase linearly with $M_{\text {wet. }}$. The slopes of $\mathrm{n}$ at 0.4 and $0.6 \mathrm{THz}$ and those of $\kappa$ at 0.4 and $0.6 \mathrm{THz}$ are also the same, respectively. This behavior is in a good agreement with the complex refractive index spectrum of polyamide (PA) immersed in water reported by Jördens et al. [18] even though PA absorbs more water than EP and saturates at about 10 mass $\%$.

\subsection{EP immersed in sulfuric acid solution}

In addition to water, sulfuric acid solutions are also known to penetrate into the EP network [4-6]. Therefore, EP specimens after immersion in the sulfuric acid solution and after drying are studied gravimetrically and spectroscopically. Up to about 150 hours, $M_{\text {wet }}$ increases linearly with square root of immersion time (see Figure 5) as the same tendency as the specimens immersed in water shown in Figure 2. After about 150 hours (6.3 days), the $M_{\text {wet }}$ becomes constant due to saturation with water and sulfuric acid. On the other hand, the mass after drying in air at $50{ }^{\circ} \mathrm{C}$ decreases to some extent but $m_{\text {dry }}$ does not return to $m_{0}$. $M_{\text {dry }}$ also increases linearly with square root of immersion time and becomes constant after 150 hours. While water molecules evaporate completely from EP network through the drying process, sulfuric acid remains due to its much lower volatility. Therefore, $M_{\text {dry }}$ indicates the sulfuric acid mass fraction $M_{\mathrm{S}}$ in the specimen.

The EDS mapping of the sulfur element for the cross section of the specimen is shown in Figure 6. This EP specimen was immersed in the sulfuric acid solution for 84 hours. The image represents that sulfuric acid penetrates into the specimen and distribute

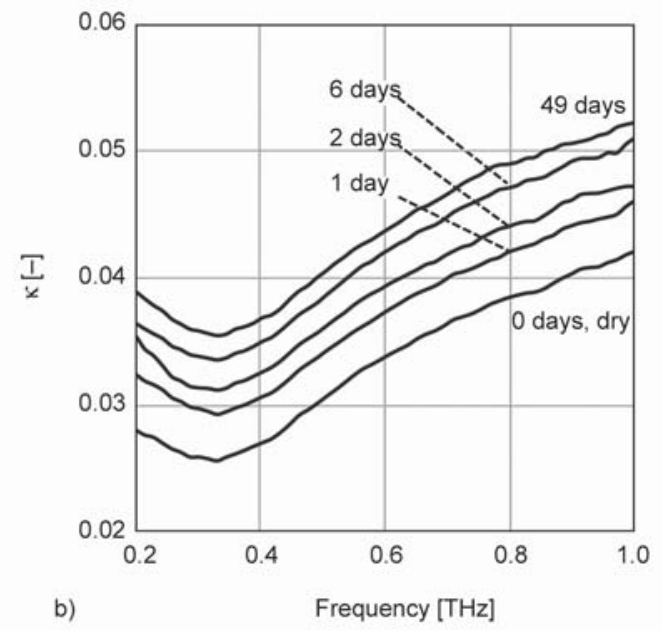

Figure 3. (a) The real and (b) imaginary parts of the complex refractive index for the EP specimen after immersion in water 

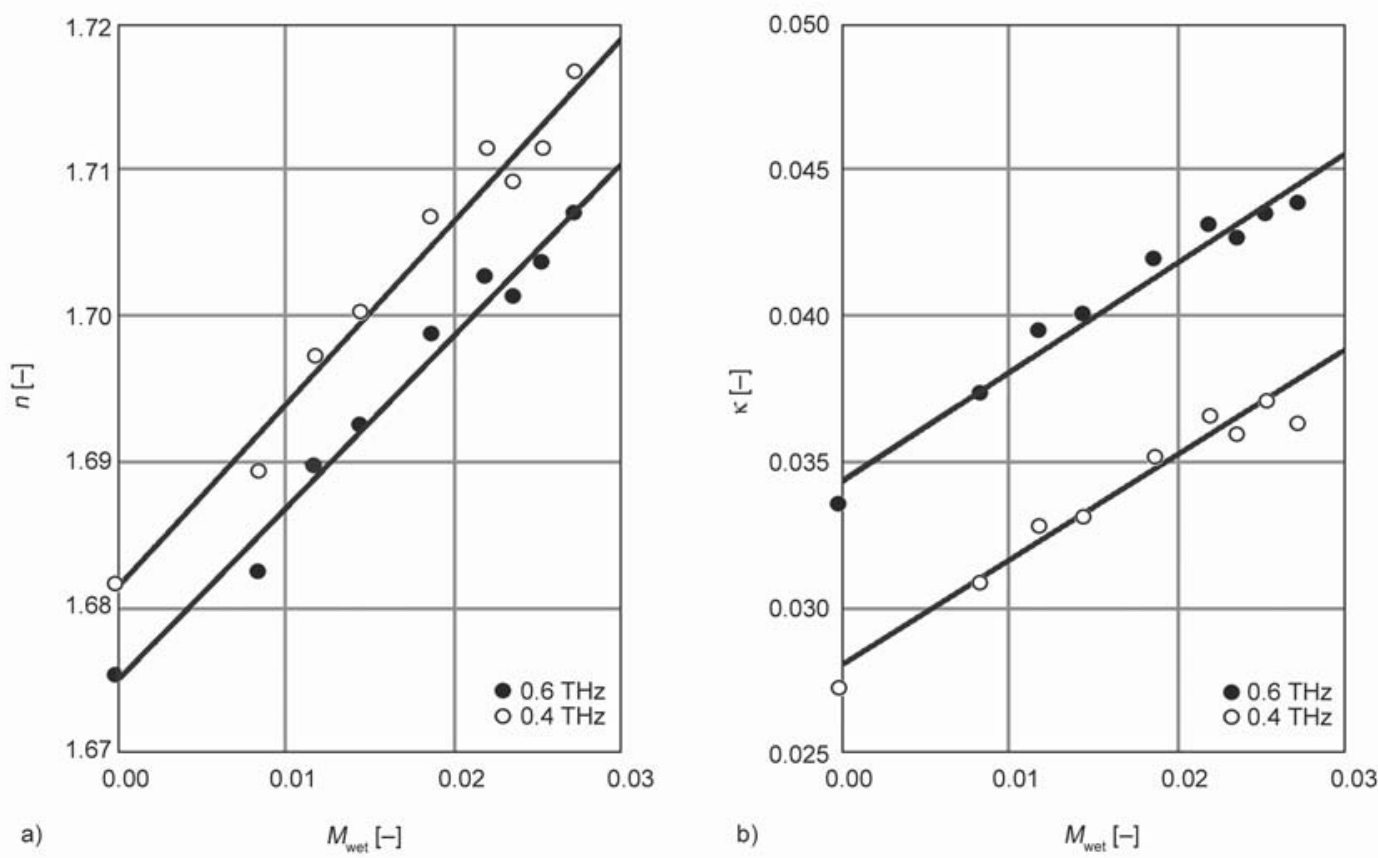

Figure 4. (a) The real and (b) imaginary parts of the complex refractive index at 0.4 and $0.6 \mathrm{THz}$ for the EP specimen with water mass fraction

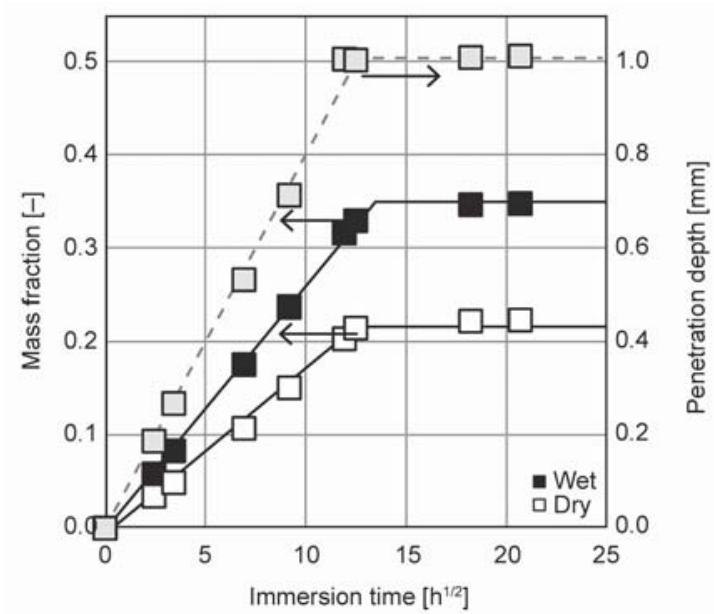

Figure 5. The wet and dry mass fractions of the EP specimen after immersion times in $2.0 \mathrm{M}$ sulfuric acid solution. In addition, gray plots are penetration depth of sulfuric acid in the specimen measured by EDS (see Figure 6)

uniformly from both sides up to $0.7 \mathrm{~mm}$ depth. The depth with square root of immersion time is also shown in Figure 5. The depth also increases linearly with square root of time and leveled off at 150 hours because the acid penetrates to whole thickness at this immersion time. These observations are consistent with other reports [4-6]. In addition, epoxy resin itself scarcely changes in respect of the molecular structure or chemically degrade despite the penetration of sulfuric acid [5].

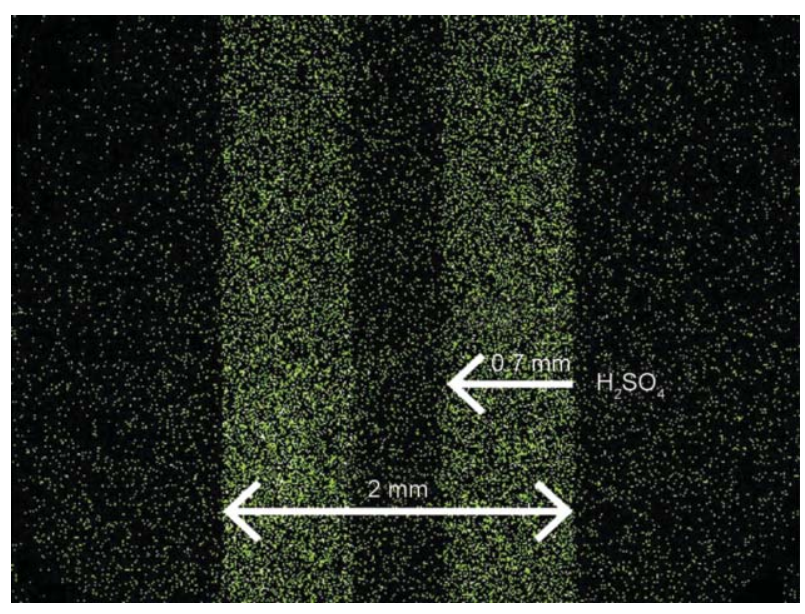

Figure 6. The EDS mapping of sulfur element at the cross section of the EP specimen ( $2 \mathrm{~mm}$ thick) after immersion in $2.0 \mathrm{M}$ sulfuric acid solution for 84 hours. Sulfur element shows that sulfuric acid penetrates into the specimen from both surfaces and reached to $0.7 \mathrm{~mm}$ depth.

The $n$ and $\kappa$ spectra of the 'wet' specimens soon after removal from the sulfuric acid solution are shown in Figure 7. Although both spectra display increase with immersion time, the $\mathrm{n}$ spectra show almost parallel lines or constant increment at all frequencies. On the other hand, the increment of $\kappa$ at higher frequency is larger than that of lower frequencies. Figure 8 shows $n$ and $\kappa$ spectra of the 'dry' specimens which are immersed in the solution and subsequently dried. Again, 

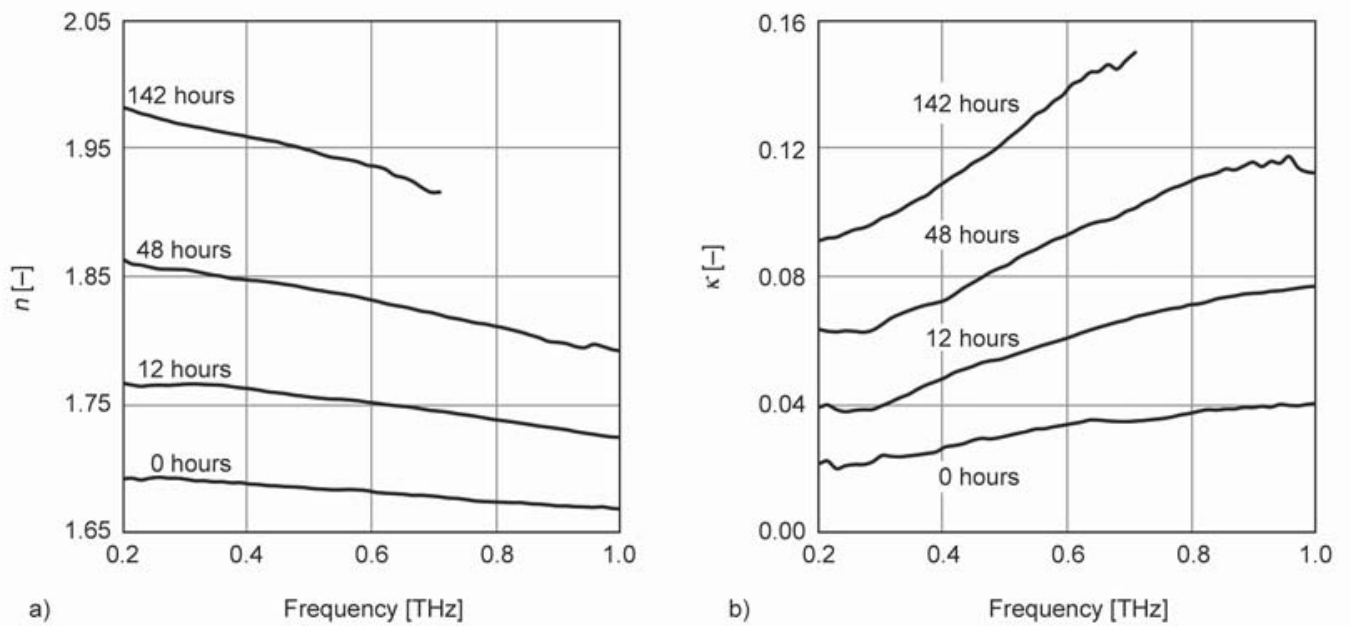

Figure 7. (a) The real and (b) imaginary parts of the complex refractive index of the EP specimen soon after removal from the $2.0 \mathrm{M}$ sulfuric acid solution
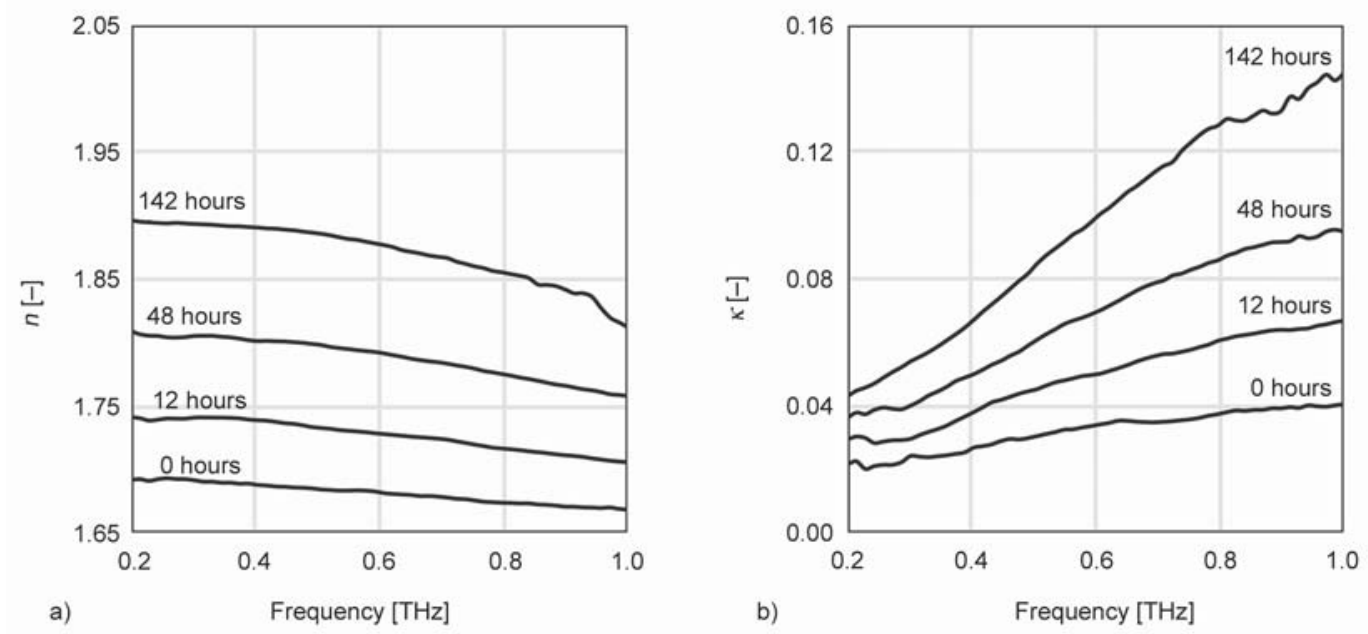

Figure 8. (a) The real and (b) imaginary parts of the complex refractive index of the EP specimen measured after immersion in the $2.0 \mathrm{M}$ sulfuric acid solution and subsequent drying in air $50^{\circ} \mathrm{C}$

the $\kappa$ at higher frequency displays larger increments and $n$ does not show this tendency. This can be seen more clearly in Figure 9, where $\kappa$ at 0.4 and $0.6 \mathrm{THz}$ are plotted against $M_{\text {dry }}$. The figure demonstrates linear dependence with the same slope of $n$ and with different slopes of $\kappa$ at 0.4 and $0.6 \mathrm{THz}$. Such behavior is observed for sulfuric acid aqueous solutions with different concentrations [33]. These results indicate that penetrated sulfuric acid causes larger increase of the $\kappa$ at the higher frequencies.

\section{Discussion}

As discussed in section 3.1, the $\tilde{n}$ of the EP specimen increased with the $M_{\text {wet }}$. Although the THz spectra of the $\tilde{n}$ of bulk water are already known $[18,23,24$, 34 ], as mentioned in section 1 , it is challenging to separate experimentally the contributions from bound and free water in materials $[18,20]$. In our study, some water molecules in the specimen can be bound to the amine and hydroxyl groups in the resin. As a result, the water state could be different from that of bulk water. This behavior is consistent with the trends observed for water immersed polyamide (PA) specimens reported by Jördens, et al. [18]

However, the $\tilde{n}$ of the epoxy specimen immersed in the sulfuric acid solution showed different changes from that immersed in water, especially for the $\kappa$ at the higher frequencies. The $\tilde{n}$ increased with the fraction of the sulfuric acid in the specimen. As mentioned in section 3.2, the sulfuric acid does not cause the chemical decomposition of the epoxy network by hydrolysis and other reactions [5]. From these results, it is guessed that the change of the $\tilde{n}$ can be attributed mainly to the sulfuric acid in epoxy resin. The 

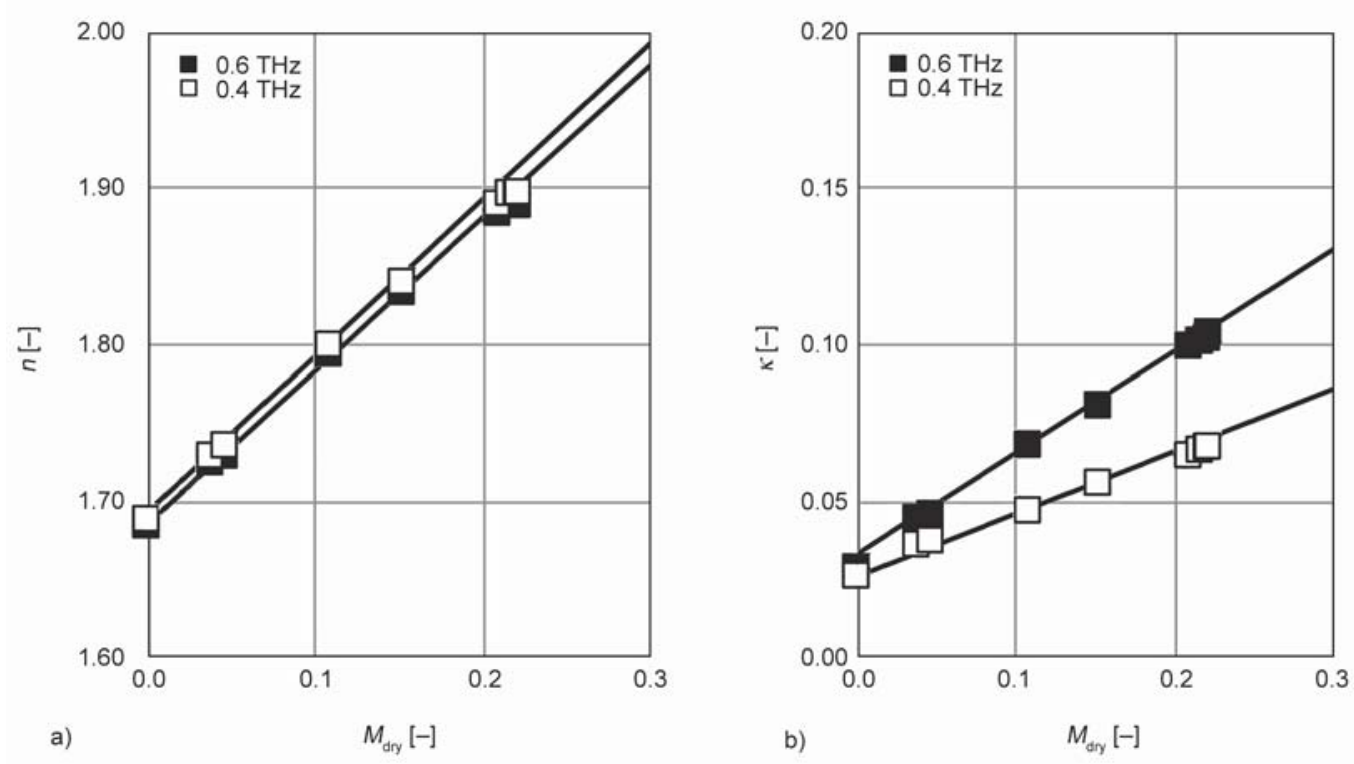

Figure 9. (a) The real and (b) imaginary parts of the complex refractive indexes at 0.4 and $0.6 \mathrm{THz}$ with the mass fraction of sulfuric acid for the EP specimen, which were measured after immersion in the $2.0 \mathrm{M}$ sulfuric acid solution and subsequent drying in air $50^{\circ} \mathrm{C}$.

lager $\kappa$ at the higher frequencies can be attributed to the sulfuric acid spectral band above $1.0 \mathrm{THz}$ with the tail extending to $0.2 \mathrm{THz}$. To understand this in more detail, the measurement at higher $\mathrm{THz}$ frequencies and with thinner samples are necessary (not a subject of the present work).

Since the increments of the $\tilde{n}$ in Figure 7 were caused by both water and acid, it is estimated that the increment $\Delta n$ and $\Delta \kappa$ are decided by a linear equation of water and acid mass fractions in EP independently. Here, $I_{\mathrm{n}, \mathrm{W}}$ and $I_{\mathrm{n}, \mathrm{S}}$ are the corresponding slopes from Figures $4 \mathrm{a}$ and $9 \mathrm{a}$, where subscripts $W$ and $S$ indicate water and sulfuric acid, respectively. While water mass fraction in EP specimen $M_{\mathrm{W}}$ is the difference between $M_{\text {wet }}$ and $M_{\text {dry }}$, sulfuric acid mass fraction $M_{\mathrm{S}}$ is $M_{\text {dry. }}$ The linear equation of $\Delta n$ is described as Equation (3):

$$
\Delta n_{\omega}=I_{\mathrm{n}, \mathrm{W}, \omega} M_{\mathrm{W}}+I_{\mathrm{n}, \mathrm{S}, \omega} M_{\mathrm{S}}
$$

where the subscript $\omega$ indicates frequency. Likewise, $\Delta \kappa$ is described as Equation (4):

$$
\Delta \kappa_{\omega}=I_{\kappa, \mathrm{W}, \omega} M_{\mathrm{W}}+I_{\kappa, \mathrm{S}, \omega} M_{\mathrm{S}}
$$

Figure 10 represents comparison between the increments by measuring with THz-TDS (Figure 7) and by calculating with the linear Equations (3) and (4) at $0.4 \mathrm{THz}$. Both the measured and calculated $\Delta n_{0.4}$ and $\Delta \kappa_{0.4}$ increase with $M_{\text {wet }}$. While the calculated $\Delta \kappa_{0.4}$ agrees well with the measured $\Delta \kappa_{0.4}$, the calculated $\Delta n_{0.4}$ is higher than the measured $\Delta n_{0.4}$. These behaviors are observed at other frequencies. The difference between calculated and measured $\Delta \mathrm{n}_{0.4}$ may be attributed to free and bound water in the specimen because $\mathrm{THz}$ radiation is sensitive to both water states [18-26]. Since amine cured EP resins have some hydrophilic groups such as hydroxyl groups and amine groups, water becomes bound to such groups to some extent. On the other hand, sulfuric acids react with amine groups in EP resins and form amine salt $[4,5]$. Therefore, the state of water in the sulfuric acid immersed specimen may differ from that in the water immersed specimen so that calculated and measured $\Delta n_{0.4}$ does not show agreement with each other. THz-TDS and some complementary analyses such as FT-IR [10, 30, 31], NMR [32], thermomechanical analysis $[31,32]$ could help to determine penetrant states in the EP resin. Nevertheless, it is confirmed that $\mathrm{THz}$ spectroscopy is a promising method to evaluate sulfuric acid in epoxy resin.

\section{Conclusions}

The $\tilde{n}$ of the EP specimens were measured by THzTDS after immersion in water and sulfuric acid solutions. It was found that $n$ and $\kappa$ depended linearly with water and sulfuric acid mass fraction in specimens, and $\Delta \kappa$ of sulfuric acid immersed specimens 

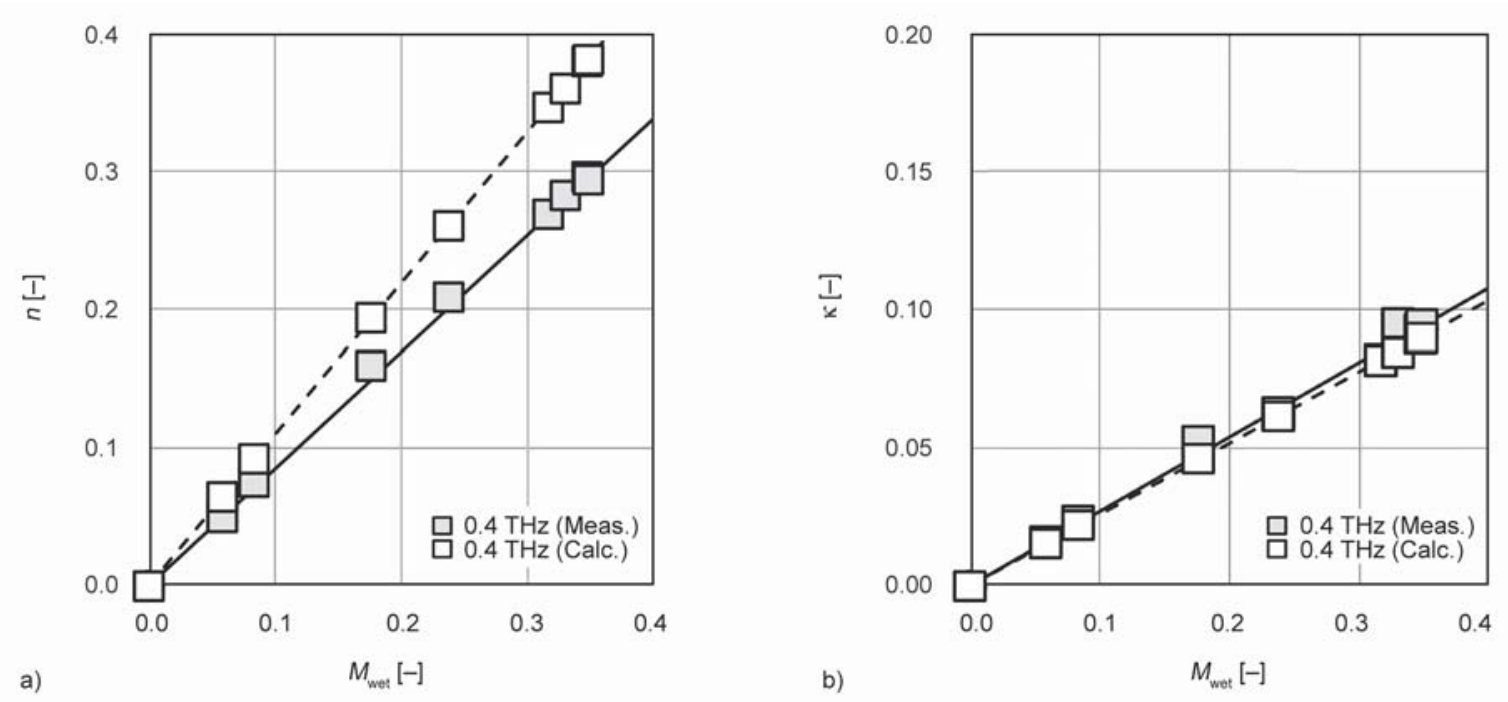

Figure 10. The increments of (a) the real and (b) imaginary parts of the complex refractive indexes at 0.4 THz: comparison between calculation by the linear equations (white plots) and the measurement by THz-TDS (gray plots)

was lager at higher frequency. THz-TDS can help to discern whether the specimen contains sulfuric acid. The increments of the $\tilde{n}$ were described with linear equations involving water and sulfuric acid mass fractions. As for $\Delta \kappa$, good agreement between calculation and measurement was observed. On the other hand, calculated $\Delta n$ was higher than the measured $\Delta n$. The difference may be attributed to free and bound water in the specimen. Therefore, THz-TDS can help to find out penetrant states in the EP resin, making it a promising NDT method to evaluate the penetration of the corrosive chemicals into resin coatings.

\section{References}

[1] Weldon D. G.: Failure analysis of paints and coatings revised edition. Wiley, New York (2009).

[2] Maggana C., Pissis P.: Water sorption and diffusion studies in an epoxy resin system. Journal of Polymer Science Part B: Polymer Physics, 37, 1165-1182 (1999). DOI: 10.1002/(SICI)1099-0488(19990601)37:11<1165 $\because$ AID-POLB11>3.0.CO;2-E

[3] Wong T. C., Broutman L. J.: Water in epoxy resins part II. Diffusion mechanism. Polymer Engineering and Science, 25, 529-534 (1985).

DOI: $10.1002 /$ pen.760250904

[4] Tsuda K., Kubouchi M.: Decomposition of polymeric materials. Zairyo-to-Kankyo, 44, 104-111 (1995). DOI: $10.3323 /$ jcorr1991.44.104

[5] Sembokuya H., Negishi Y., Kubouchi M., Tsuda K.: Corrosion behavior of epoxy resin cured with different amount of hardener in corrosive solutions. Journal of the Society of Materials Science, 9, 230-234 (2003). DOI: 10.2472/jsms.52.9Appendix_230
[6] Nakayama M., Hosokawa Y., Muraoka Y., Katayama T.: Life prediction under sulfuric acid environment of FRP using X-ray analysis microscope. Journal of Materials Processing Technology, 155-156, 1558-1563 (2004).

DOI: $10.1016 /$ j.jmatprotec.2004.04.252

[7] Amaro A. M., Reis P. N. B., Neto M. A., Louro C.: Effect of different acid solutions on glass/epoxy composites. Journal of Reinforced Plastics and Composites, 32, 1018-1029 (2013).

DOI: $10.1177 / 0731684413483886$

[8] Yochev B., Kutzarov S., Ganchev D., Staykov K.: Investigation of ultrasonic properties of hydrophilic polymers for dry-coupled inspection. in 'Proceeding of The European Conference on Non-Destructive Testing. Berlin, Germany', pp. 1-10 (2006).

[9] Silverstein R. M., Webster F. X., Kiemle D. J., Bryce D. L.: Spectrometric identification of organic compounds. Wiley, New York (1998).

[10] Kusanagi H., Yukawa S.: Fourier transform infra-red spectroscopic studies of water molecules sorbed in solid polymers. Polymer, 35, 5637-5640 (1994). DOI: $10.1016 / \mathrm{S} 0032-3861(05) 80037-0$

[11] Harrick N. J., Carlson A. I.: Internal reflection spectroscopy: Validity of effective thickness equations. Applied Optics, 10, 19-23 (1971). DOI: $10.1364 / \mathrm{AO} .10 .000019$

[12] Chen J., Gardella J. A.: Quantitative ATR FT-IR analysis of surface segregation of polymer blends of polystyrene/poly(dimethylsiloxane)-co-polystyrene. Applied Spectroscopy, 52, 361-366 (1998). DOI: $10.1366 / 0003702981943824$

[13] Wietzke S., Jansen C., Reuter M., Jung T., Kraft D., Chatterjee S., Fischer B. M., Koch M.: Terahertz spectroscopy on polymers: A review of morphological studies. Journal of Molecular Structure, 1006, 41-51 (2011). DOI: $10.1016 /$ j.molstruc.2011.07.036 
[14] Wietzke S., Jansen C., Jung T., Reuter M., Baudrit B., Bastian M., Chatterjee S., Koch M.: Terahertz time-domain spectroscopy as a tool to monitor the glass transition in polymers. Optics Express, 17, 19006-19014 (2009).

DOI: 10.1364/OE.17.019006

[15] Pastorelli G., Trafela T., Taday P. F., Portieri A., Lowe D., Fukunaga K., Strlič M.: Characterisation of historic plastics using terahertz time-domain spectroscopy and pulsed imaging. Analytical and Bioanalytical Chemistry, 403, 1405-1414 (2012).

DOI: $10.1007 / \mathrm{s} 00216-012-5931-9$

[16] Rutz F., Koch M., Khare S., Moneke M., Richter H., Ewert U.: Terahertz quality control of polymeric products. International Journal of Infrared and Millimeter Waves, 27, 547-556 (2007).

DOI: $10.1007 / \mathrm{s} 10762-006-9106-7$

[17] Macutkevic J., Seliuta D., Valusis G., Adomavicius R., Kuzhir P., Paddubskaya A., Shuba M., Maksimenko S., Coderoni L., Micciulla F., Sacco I., Bellucci S.: Terahertz time domain spectroscopy of epoxy resin composite with various carbon inclusions. Chemical Physics, 404, 129-135 (2012).

DOI: 10.1016/j.chemphys.2012.02.002

[18] Jördens C., Wietzke S., Scheller M., Koch M.: Investigation of the water absorption in polyamide and wood plastic composite by terahertz time-domain spectroscopy. Polymer Testing, 29, 209-215 (2010). DOI: $10.1016 /$ j.polymertesting.2009.11.003

[19] Nagai N., Kumazawa R., Fukasawa R.: Direct evidence of inter-molecular vibrations by $\mathrm{THz}$ spectroscopy. Chemical Physics Letters, 413, 495-500 (2005). DOI: $10.1016 /$ j.cplett.2005.08.023

[20] Federici J. F.: Review of moisture and liquid detection and mapping using Terahertz imaging. Journal of Infrared, Millimeter, and Terahertz Waves, 33, 97-126 (2012).

DOI: $10.1007 / \mathrm{s} 10762-011-9865-7$

[21] Inagaki T., Ahmed B., Hartley I. D., Tsuchikawa S., Reid M.: Simultaneous prediction of density and moisture content of wood by terahertz time domain spectroscopy. Journal of Infrared, Millimeter, and Terahertz Waves, 35, 949-961 (2014). DOI: $10.1007 / \mathrm{s} 10762-014-0095-7$

[22] Nagai M., Yada H., Arikawa T., Tanaka K.: Terahertz time-domain attenuated total reflection spectroscopy in water and biological solution. International Journal of Infrared and Millimeter Waves, 27, 505-515 (2006). DOI: 10.1007/s10762-006-9098-3

[23] Yada H., Nagai M., Tanaka K.: Origin of the fast relaxation component of water and heavy water revealed by terahertz time-domain attenuated total reflection spectroscopy. Chemical Physics Letters, 464, 166-170 (2008).

DOI: 10.1016/j.cplett.2008.09.015
[24] Rønne C., Åstrand P-O., Keiding S. R.: THz Spectroscopy of liquid $\mathrm{H}_{2} \mathrm{O}$ and $\mathrm{D}_{2} \mathrm{O}$. Physical Review Letters, 82, 2888-2891 (1999).

DOI: 10.1103/PhysRevLett.82.2888

[24] Vinh N. Q., Sherwin M. S., Allen S. J., George D. K., Rahmani A. J., Plaxco K. W.: High-precision gigahertzto-terahertz spectroscopy of aqueous salt solutions as a probe of the femtosecond-to-picosecond dynamics of liquid water. The Journal of Chemical Physics, 142, 164502/1-164502/7 (2015).

DOI: $10.1063 / 1.4918708$

[26] Koeberg M., Wu C-C., Kim D., Bonn M.: THz dielectric relaxation of ionic liquid:water mixtures. Chemical Physics Letters, 439, 60-64 (2007). DOI: 10.1016/j.cplett.2007.03.075

[27] Hochrein T.: Markets, availability, notice, and technical performance of Terahertz systems: Historic development, present, and trends. Journal of Infrared, Millimeter, and Terahertz Waves, 36, 235-254 (2015).

DOI: 10.1007/s10762-014-0124-6

[28] Hangyo M., Nagashima T., Nashima S.: Spectroscopy by pulsed Terahertz radiation. Measurement Science and Technology, 13, 1727-1738 (2002). DOI: 10.1088/0957-0233/13/11/309

[29] Watanabe M., Kuroda S., Yamawaki H., Shiwa M.: Terahertz dielectric properties of plasma-sprayed thermalbarrier coatings. Surface and Coatings Technology, 205, 4620-4626 (2011).

DOI: $10.1016 /$ j.surfcoat.2011.03.144

[30] Kondo T., Sawatari C.: A Fourier transform infra-red spectroscopic analysis of the character of hydrogen bonds in amorphous cellulose. Polymer, 37, 393-399 (1996).

DOI: $10.1016 / 0032-3861(96) 82908-9$

[31] Ping Z. H., Nguyen Q. T., Chen S. M., Zhou J. Q., Ding Y. D.: States of water in different hydrophilic polymers - DSC and FTIR studies. Polymer, 42, 8461-8467 (2001). DOI: 10.1016/S0032-3861(01)00358-5

[32] Hatakeyema T., Yamauchi A., Hatakeyema H.: Studies on bound water in poly(vinyl alcohol). Hydrogel by DSC and FT-NMR. European Polymer Journal, 20, 6164 (1984). DOI: 10.1016/0014-3057(84)90223-4

[33] Kusano M., Aoki S., Kubouchi M.: Evaluation of acid and alkali aqueous solution penetration in polymeric materials by Terahertz time-domain spectroscopy. in 'The $11^{\text {th }}$ European Conference on Non-Destructive Testing 2014. Prague, Czech Republic' p.9 (2014).

[34] Rungsawang R., Ueno Y., Ajito K.: Detecting a sodium chloride ion pair in ice using terahertz time-domain spectroscopy. Analytical Sciences, 7, 917-920 (2007). DOI: 10.2116/analsci.23.917 